\title{
The evolution from e(lectronic)Maintenance To i(ntelligent)Maintenance
}

\author{
Diego Galar, Karina Wandt, Ramin Karim \\ Division of Operation and Maintenance Engineering \\ Luleå University of Technology \\ Luleå, Sweden \\ Tel. +46920492437 \\ Fax + 46920491935 \\ diego.galar@ltu.se \\ Luis Berges \\ Department of Design Engineering and Manufacturing \\ University of Zaragoza \\ Zaragoza, Spain
}

\begin{abstract}
iMaintenance stands for integrated, intelligent and immediate maintenance. It integrates various maintenance functions and connects these to all devices, using advanced communication technologies. The main challenge is to integrate the disparate systems and capabilities developed under current eMaintenance models and to make them immediately accessible through intelligent computing technologies. iMaintenance systems are computer-based, able to evolve with the system that they monitor and control, and they can be embedded in the system's components, providing the ability to integrate new functionality with no downtime. This article will show how iMaintenance systems can provide decision-making support, thereby going beyond merely connecting various maintenance systems.
\end{abstract}

\section{Introduction}

Maintenance teams are responsible for maintaining the operation of a system based on the observations of the system's users or on messages originating from test devices built into the equipment to establish failure diagnostics, to identify and locate system equipment failures, and if required, to replace a failed item, in part or in full. The gathering of data, messages and observations serving to track down equipment failures is called maintenance management ${ }^{(1)}$. The architecture of complex systems is, in general, based on information transfers between electronic equipment linked by a communication network. The equipment can be difficult to access; various items can be from ten centimetres to several hundred metres away ${ }^{(2)}$.

Generally speaking, management information systems monitor and record messages and observations in connection with industrial processes. These systems often are reactive in that they respond to present levels of monitored parameters; at most, they control the generation of alarms when a parameter exceeds pre-set values or threatens to do so. A 
typical process control system monitors sensed parameters to ensure they remain within pre-set limits. Often present levels can be displayed graphically to highlight trends ${ }^{(3)}$.

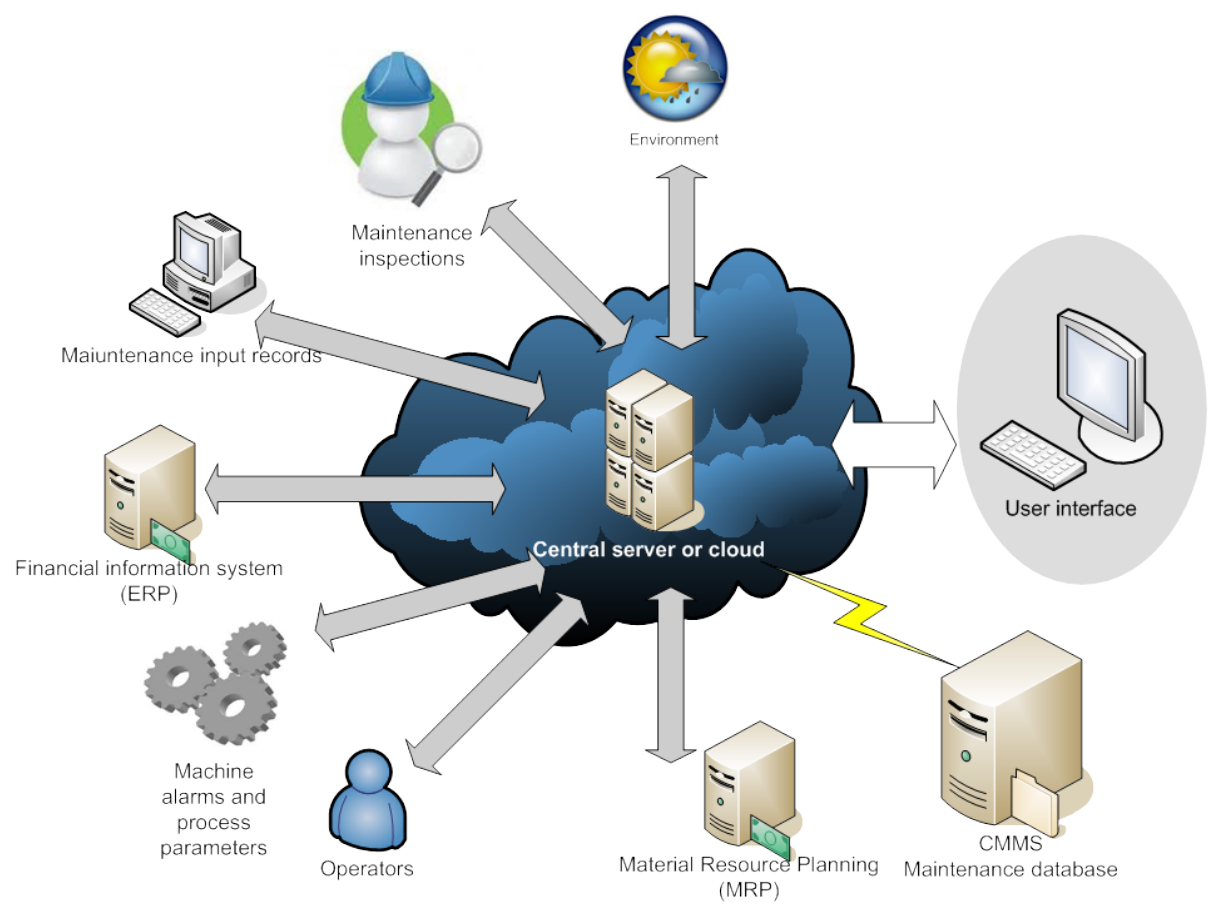

Figure 1. Existing interfaces to collect relevant information for maintenance

Other management information systems can schedule maintenance procedures. By defining the useful life of each item among a number of related or inter-dependent items, it is possible to schedule repair, replacement or preventive maintenance operations more efficiently so as to minimize downtime. The idea is to plan replacement or repair for as late as practicable before an actual failure, preferably using intelligent scheduling procedures to minimize downtime. The scheduling system prompts or warns plant personnel to attend to each item which may need attention at or soon after the time at which its maintenance becomes critically important.

It would be advantageous to provide an integrated system which monitors various assets of plant equipment and also accounts for the interdependence of the subsystems, makes decisions or predictions in view of stored design criteria, and makes all this information available to plant personnel. Therefore, the interrelations of various items or subsystems, their design specifications, their history and their current conditions should be considered when assessing operational conditions and maintenance needs, or when evaluating operations on an engineering level ${ }^{(4)}$.

It is generally advisable for maintenance personnel to collect all available data regarding the subsystems operating in a plant or in an area of the plant, to coordinate maintenance and repair activities. If this is done, downtime for work on one or more articles or subsystems can be used for simultaneous work on others. However, comprehensive calculation and analysis of relevant plant conditions can be lengthy and costly. In a monitoring system information on operational conditions may only be immediately available to the operators and maintenance technicians, but the engineers and managers 
must collect and analyse much of the same information to plan their activities. Each group tends to collect and analyse data in a manner best suited to its own area of concern ${ }^{(5)}$.

An integrated arrangement is certainly more efficient and useful than one in which the various departments operate substantially independent information systems. The present paper addresses the integration of diagnostic instrumentation to perform more effective and efficient maintenance.

The maintenance management system and the process control system can be integrated with instrument data collection from a variety of sources. Various condition parameters can be factored into an integrated diagnostics system with technical specifications and historical data for condition based maintenance and aging management ${ }^{(6)}$. This integration requires the creation of a common taxonomy to select information, create links among data sources and extract further knowledge, so that operation and maintenance decisions can be made more effectively from a greater base of knowledge.

eMaintenance needs to be extended from principally focus on the provision of information, to a dimension with focus on processing and the provision of decisions ${ }^{(7)}$. The contribution of human intelligence is an important aspect within processing and decision-making. Artificial intelligence (AI), which may be defined as the ability of a system to perform intellectual tasks associated with human intelligence (e.g. to derive a decision by using reasoning, generalizing and experience-based knowledge) ${ }^{(8)}$, can be considered as an essential enabler that complement existing provision of information services and contribute to increased satisfaction of the service consumer. Hence, adapting to new situations is an important characteristic of intelligent systems. However, this ability requires information input that is received from systems, which in turn also should be intelligent. The required intelligence will probably always be a combination of human and artificial intelligence, even though the latter part is expected to increase in importance ${ }^{(9)}$.

\section{2. eMaintenance used to solve ICT issues}

Maintenance is defined by the European Committee for Standardization (EN 13306:2001) ${ }^{(10)}$ as the combination of all technical, administrative and managerial actions during the life cycle of an item intended to retain it in, or restore it to, a state in which it can perform the required function or a combination of functions of an item which are considered necessary to provide a given service. Once determined, maintenance objectives or priorities, strategies, and responsibilities are implemented by means of maintenance planning, maintenance control and supervision.

Maintenance Management includes corrective, preventive and proactive maintenance, inventory and procurement, work order systems, computerized maintenance management systems (CMMS), reliability centred maintenance, total productive maintenance, financial optimization, technical training, and continuous improvement.

From an organizational point of view, maintenance management must align actions at three levels of business activities. According to ${ }^{(11)}$, this means the following: 
- The strategic level establishes maintenance priorities in accordance with business goals. These goals are materialized by a maintenance plan which will establish critical targets in current operations. In addition, maintenance management at this level is responsible for determining the skills and technology necessary to improve maintenance effectiveness and efficiency. At this stage, connectivity with systems like ERP (Enterprise Resource Planning) where financial data or human resourced data are located is essential.

- The tactical level determines the assignment of maintenance resources to fulfil the maintenance plan according to the production plan dictated by the MRP system. Detailed maintenance requirements, planning and scheduling are established at this level. The tactical level is mainly supported by EAM (Enterprise Asset Management) which contains information on all assets, not just the productive ones ${ }^{(12)}$. Within EAM, the role of CMMS is especially relevant; a natural maintenance data repository, it performs scheduling, planning and resource allocation in terms of the labour force and spare parts.

- The operational level ensures that maintenance tasks are carried out by skilled technicians, in the time scheduled, following the correct procedures, and using the proper tools. This level of maintenance management is also responsible for the recoding of data for diagnosis and/or prognosis purposes. This is arguably the most challenging level, as two different types of data are merged: CMMS and Condition Monitoring (CM) data. While CMMS and ERP deal with similar types of data stored in a similar way, CM deals with enormous amounts of online and offline data from disparate data sources like vibration, thermography or tribology analysis.

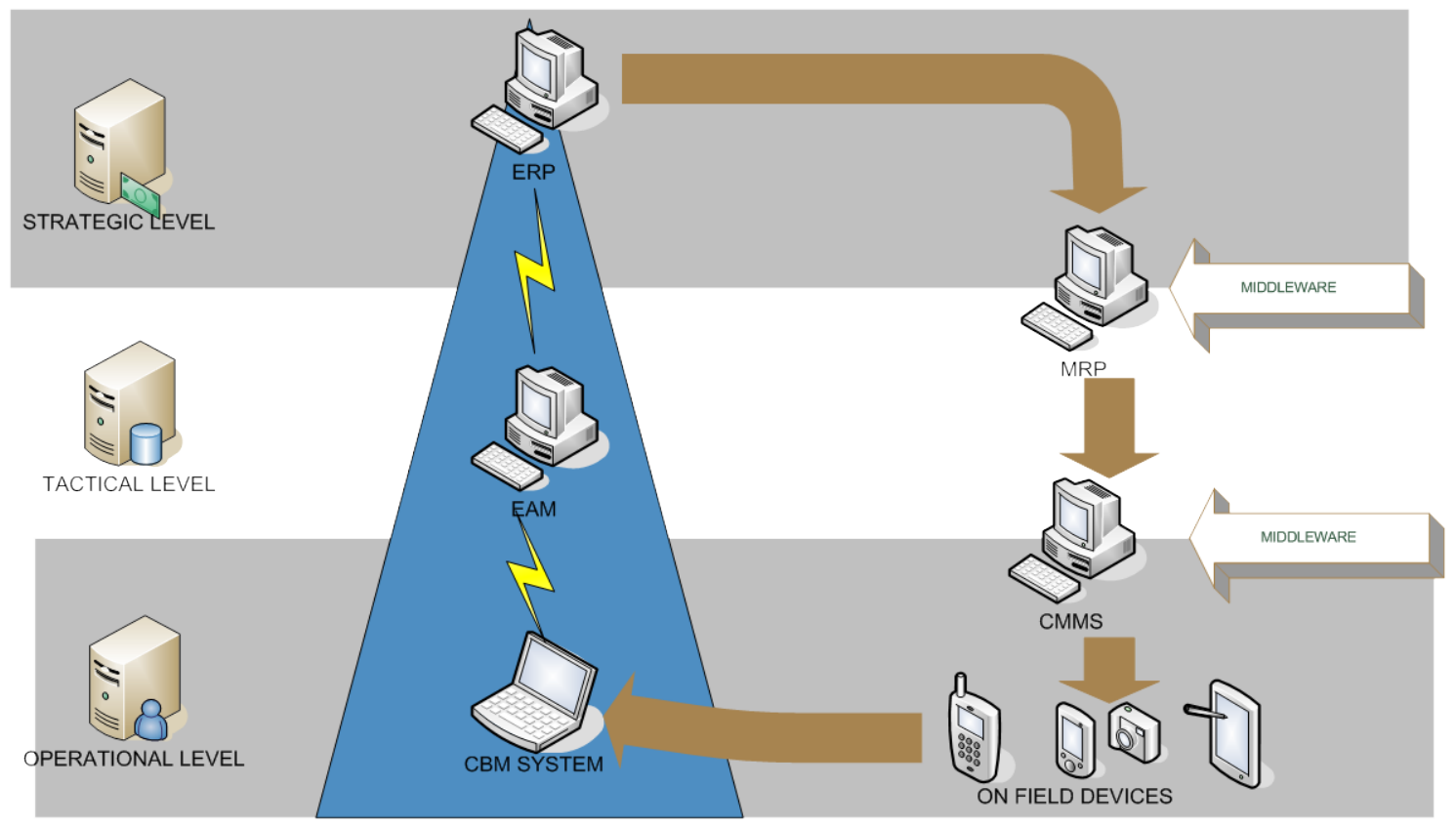

Figure 2. Levels and IT systems in the MDSS process 


\subsection{Challenges in maintenance information source fusion}

The measurements taken and analysis performed by a CM system must be available to maintenance planners who work with CMMS for the purpose of scheduling predictive and other types of work orders ${ }^{(13)}$. In the past, maintenance organizations that used both CMMS and CM technologies linked the two systems by inputting CM data manually into CMMS. While this is an acceptable way to transfer data for the purpose of scheduling predictive maintenance work orders, it is time-consuming.

Passive data exchange, a recent development, involves writing pertinent CM data to a specified local or network directory. Relevant data to be exchanged include equipment identification, date and time stamps, repair priority, repair recommendations, and observations. The CMMS program routinely checks this directory, and if a transfer file is found, CMMS reads it and imports it into the CMMS database. This method of data transfer is very specific to CM and CMMS software. The passive data transfer method is better than manual data entry but falls well short of the total automation and instant access to information that is possible when the CMMS and CM programs are fully integrated.

\subsection{Integration methodologies}

The first step to integrating CMMS and CM into an automatic system is setting up a way for the two systems to communicate. In the case of CMMS and CM technologies, consistent data must be found in each system so they can communicate using a common base of information. For example, all equipment monitored by the CM system must also exist in the CMMS database, and must be called by the same name in each system.

Next, there must be a system of data cross-references between the sensors, meter tags, or other measurement tools in a CM system and the appropriate module in CMMS that associates readings in one system with readings in the other. Meter readings or alarm triggers that are out of the acceptable range set up in CMMS should trigger a predefined work order. Any discrepancy in this cross-reference for a piece of equipment will nullify the link for that piece of equipment, making the ability to predict problems that much less comprehensive. This makes upfront planning of data entry rules and database setup a critical part of the pre-integration process.

The third step in fully integrating CMMS with CM is to provide a direct link between the systems' data tables. This is referred to as an "active exchange" of data. Today, the CMMS databases use open architectures. They can be read from and written to CM programs with certain capabilities.

Information and Communication Technology (ICT) is considered a maintenance pillar, along with maintenance engineering and organizational methods ${ }^{(13)}$. In fact, the development of ICT in the maintenance field first created what is globally called “eMaintenance," previously termed tele-maintenance by many researchers. 
Simply stated, eMaintenance integrates different maintenance-related systems into one information system, making ICT and connectivity a priority. It attempts to solve the following issues: ${ }^{(14)}$

- Miniaturization of devices, thus increasing the ways data can be acquired, including mobile systems.

- Extension of communication technologies, including wireless and usage of the Internet as a main distributed platform for business operation.

These issues have been partially solved by the development of information standards for system interoperability, such as MIMOSA and OSA-CBM ${ }^{(15)}$. These technologies have favoured research into smart sensors, devices to be carried by a maintenance crew, or more powerful wireless technologies, mainly Bluetooth, making them more robust in industrial environments than those developed for domestic purposes.

Most authors, as ${ }^{(6)}$ argue, link eMaintenance with those technologies which allow the optimization of maintenance and integrate it with other business related issues within and outside a company.

None of the various eMaintenance definitions explicitly mentions intelligence as a fundamental characteristic. Nevertheless, each definition implicitly recognizes such its importance, especially in real time data processing and the automation of some maintenance tasks. In fact, eMaintenance, according to existing literature, deals with integration, communication and interoperability to achieve real ICT data-driven maintenance management. Therefore, ICT is a fundamental dimension of maintenance; it enhances the cooperative and collaborative characteristics of maintenance activities. As such, ICT is at the base of every definition of eMaintenance.

\section{3. iMaintenance, something beyond connectivity}

In general terms, even though known frameworks recognize the need for intelligence within eMaintenance systems, none goes beyond a shallow model of that dimension. However, several industrial and academic eMaintenance platforms have been developed and are in use today. Within such platforms, artificial intelligence in general and machine learning algorithms in particular are important enablers of the modelling of maintenance tasks, such as condition monitoring, prognostics, prognosis and decision support ${ }^{(16)}$.

The various contributions to the enhancement of eMaintenance components, namely adding AI based capabilities to known platforms, are rarely holistic. Rather, they build a more or less successful montage upon a given ICT infrastructure. Intelligence is the basis of the eMaintenance concept at every business level: strategic, tactical and operational. But something is missing in current eMaintenance systems; they cannot fulfil the expectation that eMaintenance is an intelligent system able to make decisions using a holistic approach. iMaintenance can be considered as an extension of eMaintenance; eMaintenance focuses on the provision of information, while iMaintenance focuses on processing and the provision of decisions ${ }^{(7)}$. 
The whole iMaintenance system can be considered an intelligent system, a system with artificial intelligence, interacting with other constituents of an enterprise to achieve its goals $^{(17)(18)(19)}$.

\subsection{Proposed architecture}

Maintenance customers demand knowledge extraction from gathered data so that their respective industries can become competitive. The uniform service architecture offered by iMaintenance is designed to meet their needs. The iMaintenance architectures provide integration frameworks, linking standard software at management (i.e., planning) and automation (i.e., command) levels. Information technology influence is driven top down from the management level while technology influence is driven upward from the field level. This has affected the reliance and interoperability between individual software and hardware and created a need for consistent standards and user adapted solutions. eMaintenance developments have successfully found solutions for many of these needs.

Objectives of iMaintenance technology include the creation of standardized procedures and reference processes and the development of assessment models to facilitate decision making in business based maintenance. iMaintenance must define structure and implement the framework required for knowledge acquisition. Further, it must provide an integration concept for universal and global information technology support. This technology architecture should include computerized maintenance management systems (CMMS), maintenance decision support (MDSS), condition based maintenance systems (CBM), control systems, maintenance performance measurement systems (MPM), and machinery information management open systems alliance (MIMOSA) or similar, as the glue of all different pieces.

In addition, other company information systems, including enterprise resource planning (ERP), manufacturing resource planning (MRP), engineering data management (EDM), and enterprise asset management (EAM), should be considered.

Overall, iMaintenance technology is intended to provide an umbrella for methods and processes, knowledge based business processes, and knowledge based technical processes. Making use of innovative information technology solutions, it provides global, consistent and uniform information. The methods and processes offered by iMaintenance follow uniform maintenance methodologies, establish standards for globally applicable methods and reference processes, and develop assessment models for performance measurement ${ }^{(18)}$. Such development includes life cycle costs, RAMS analysis and MPM methodologies.

Knowledge based business processes must be able to implement asset management concepts, target maintenance strategy definitions and implementations, collect and consolidate existing know-how, and derive best practices from business and maintenance processes through data mining techniques. They also offer increased plant productivity, systematic utilization of know-how, modules for decision support, and performance monitoring. 


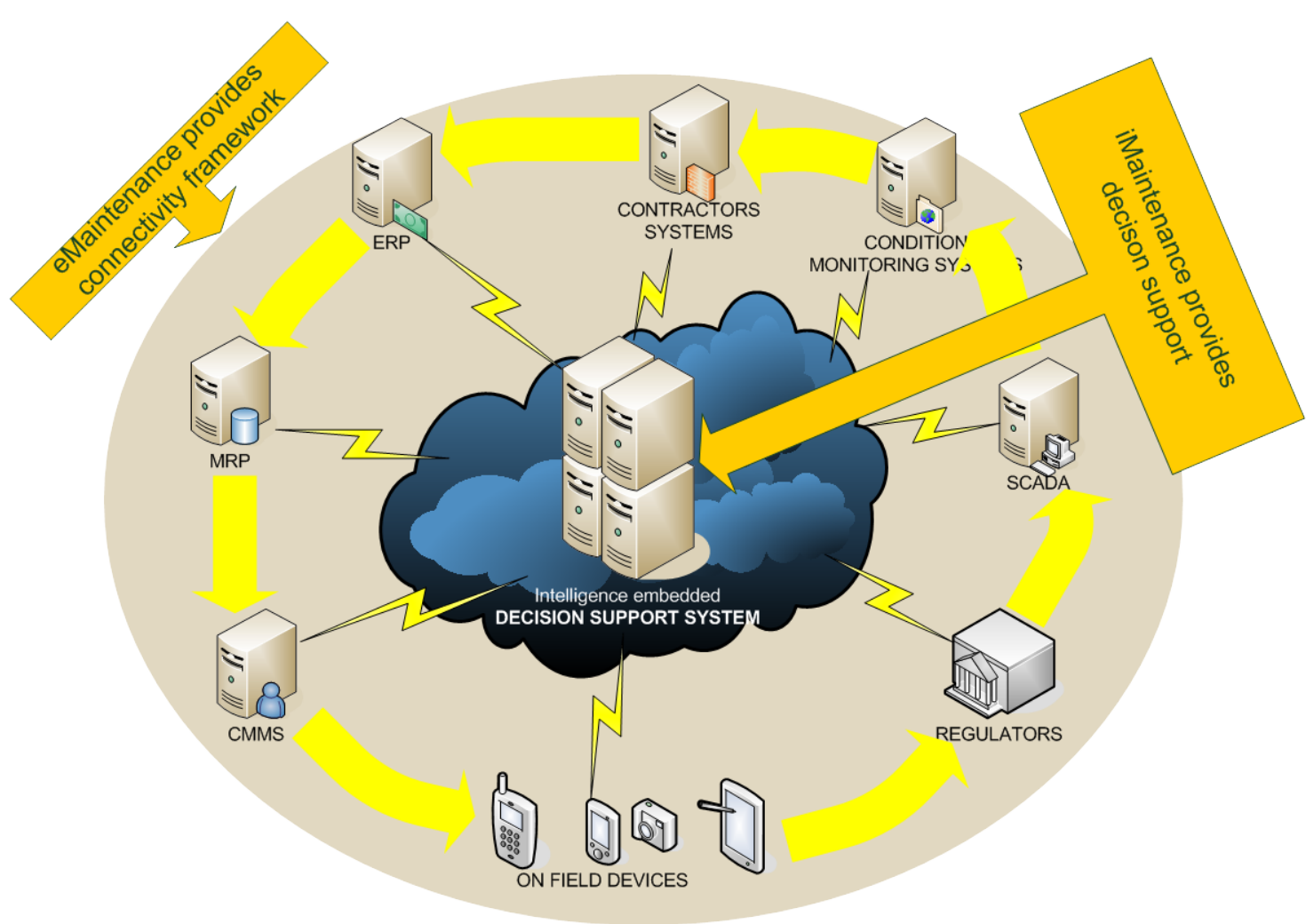

Figure 3. Combination of iMaintenance and eMaintenance concepts

Maintenance decision support methods and modules for diagnosis, optimization and residual life estimation must be included in this architecture. Numerous health prediction techniques are discussed in the literature ${ }^{(20)}{ }^{(21)}$. In addition, existing databases containing descriptions of plant assets or plant behaviour must be integrated to create consolidated eMaintenance architecture. A maintenance information model must integrate all functions, taking standards and strategic system platforms into account. Innovative technologies for teleservice and virtual support should also be provided. Finally, there must be effective mechanisms for experience exchange and knowledge management, performing data mining for rule based models and to determine best practices.

\section{MDSS using existing data: The challenge}

Two systems are used in most maintenance departments. First, Computer Maintenance Management Systems (CMMS) are the core of traditional maintenance record-keeping practices. Second, Condition Monitoring (CM) systems directly monitor asset component parameters. However, attempts to link observed CMMS events to CM sensor measurements have been fairly limited.

CMMS can perform a wide variety of functions to improve maintenance performance. In fact, it is the central organizational tool for world-class maintenance operations. Among many other critical features, CMMS can facilitate a shift in emphasis from reactive to preventive maintenance by allowing a maintenance professional to set up automatic PM work order generation. CMMS can also provide historical information 
which is used to adjust PM system setup over time to minimize unnecessary repairs, while avoiding run-to-failure repairs. PMs for a given piece of equipment can be set up on a calendar schedule or a schedule that utilizes meter readings. CMMS includes inventory tracking, workforce management, and purchasing; it stresses database integrity to safeguard vital information. The results include optimized equipment uptime, lower maintenance costs, and better plant efficiency.

While CMMS is a useful organizational tool, it cannot directly monitor equipment conditions. CM systems, however, can accurately monitor real-time equipment performance and alert the maintenance department to any changes in performance trends. A CM system can track such things as vibration, oil condition, temperature, etc. The best CM systems can analyse measurements like vibration and diagnose machine faults. Such expert system analysis puts maintenance procedures on hold until absolutely necessary, thus ensuring maximum equipment up-time. In addition, expert systems offer diagnostic fault trending where individual machine fault severity can be observed over time.

Both CMMS and CM systems have strong suits that make them indispensable to maintenance operation improvements. On the one hand, CM systems excel at monitoring equipment conditions; on the other hand, CMMS can organize overall maintenance operation. The logical conclusion is to combine the two technologies, creating a seamless system that avoids catastrophic breakdowns, but eliminates needless repairs ${ }^{(22)}$.

To operate a maintenance organization effectively and efficiently, one needs precise information combined with the ability to react quickly to impending emergencies. A combination of Computerized Maintenance Management Systems (CMMS) and Condition Monitoring (CM) technologies can help maintenance crews eliminate the vast majority of unscheduled equipment repairs. While using a good version of either technology can help an O\&M department to achieve its goals, combining the two can have exponentially more positive effects on performance than either system alone might achieve.

When the strengths of top-notch CMMS (including preventive maintenance scheduling, automatic work order generation, maintenance inventory control, and data integrity) are combined with a cutting-edge CM system (including multiple-method condition monitoring, trend tracking, and expert system diagnoses), work orders can be generated automatically, as shown in the figure below ${ }^{(23)}$. 


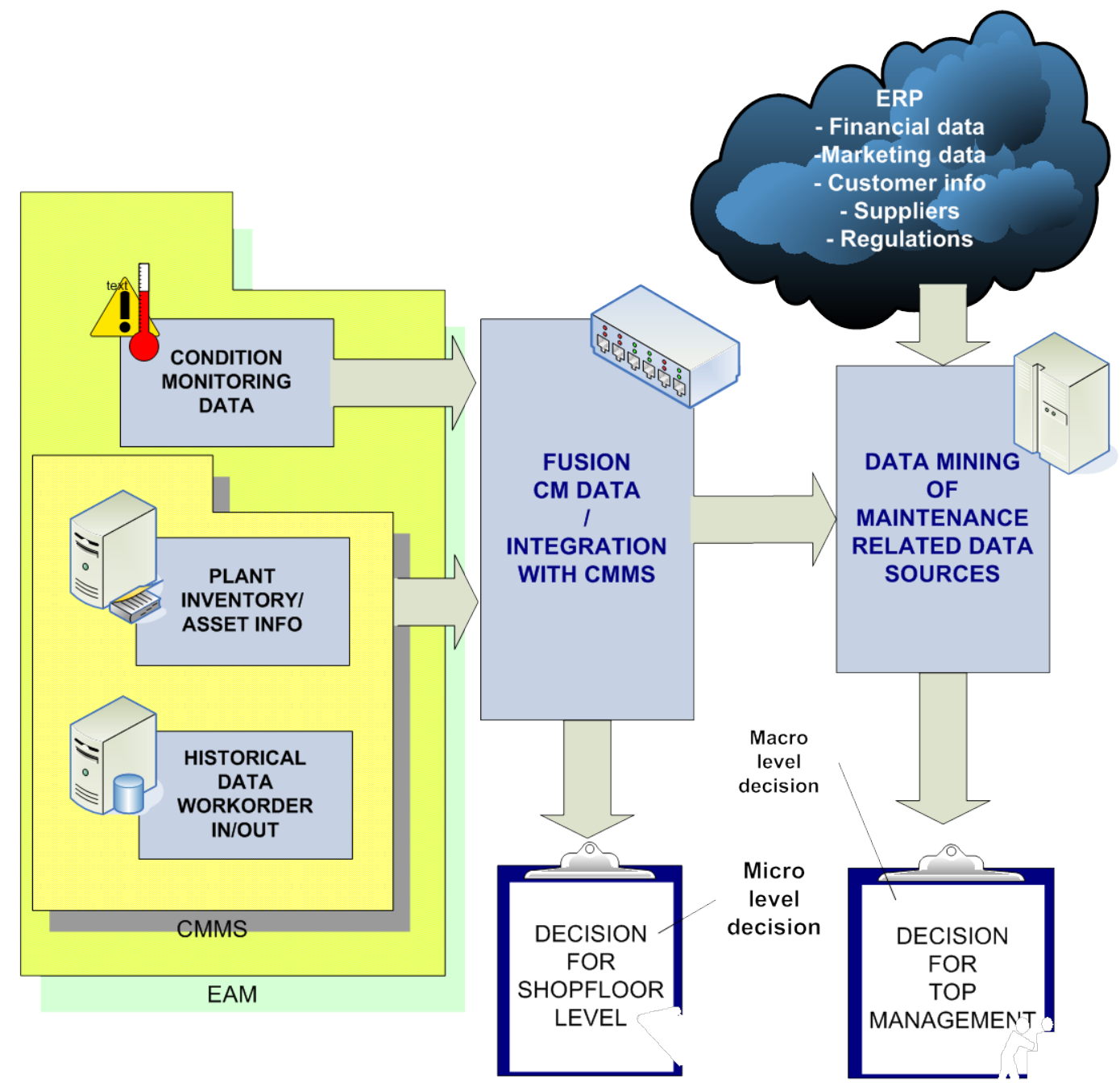

Figure 4. Decision levels as a result of maintenance data integration

The enormous possibilities are central to the predictive maintenance concept. Just a few years ago, linking CMMS and CM technology was a vision easily dismissed as infeasible or too expensive and difficult to warrant investigation. Now, the suppliers of CMMS and CM technology have made it possible to achieve such a link relatively easily and inexpensively.

Simply stated, the integration of CMMS and CM systems is feasible because of IT evolution. With the development of open communication protocols, the information accumulated by smart field devices can be captured by asset management software. It is no longer necessary for technicians to carry handheld communicators or laptops into a plant to evaluate the condition of instruments, some of which are quite inaccessible or in hazardous areas, to be followed by manually documenting test results and device status.

Current applications compile databases of every smart instrument used for process control, including design parameters, original configuration, maintenance history and current operating condition. With these online tools, technicians can obtain up-to-date 
information on any device; they do not have to make manual entries back into a system. Every event is recognized and recorded, whether initiated by a technician or caused by an external force such as equipment breakdown or power failure. This process produces an immediate result for the shop floor level: work orders are determined by devices that collect automatically information and send a warning if something goes wrong. Users can refer to recorded alerts to identify any devices that have been problematic over time and what corrective steps have been taken previously. Automated documentation provides a seamless record of events in a given production area, including communication failures, device malfunctions and process variables that are out of range. Armed with this information, maintenance personnel are better equipped to understand and resolve nagging repetitive issues to improve the process. If there is an issue, or if maintenance personnel experience a rash of issues, they can go back into the records and get a sense of what has been going on over time, searching by a specific device or by location.

Once the method of data transfer is established, the decision of which CMMS and CM systems to use is determined by the features of each data transfer and how these features interact. Generally speaking, while eMaintenance systems provide connectivity, iMaintenance systems produce new knowledge as a result of the data merging and derive useful decisions for the maintenance crew.

The ability to set up alarms in a CM program that automatically generate work orders based on pre-defined tasks in CMMS is paramount to the successful function of an integrated CMM/CM system. As the figure above shows, the first level of decisionmaking concerns shop floor level people and immediate interventions ${ }^{(23)}$. Alarms should be displayed on screens and stored; the user should be able to view records on logged alarms, acknowledged alarms, unacknowledged alarms, and recorded/unrecorded meter readings to track performance trends. This can be considered a low level of intelligence since it is the direct consequence of data integration. However, this process can produce more complex and non-evident new knowledge.

The combined system should be able to predict potential equipment failure based on measurements, historical data and past experiences. The iMaintenance system should also include an automatic diagnostic and prognostic system ${ }^{(24)(25)}$ based on logic and empirically derived from human analysts' experience. Retention of knowledge in maintenance departments is a challenge that can be addressed by the successful integration of a combined system. As historical information is not immediately evident to technicians, a combined system can make a major contribution to the daily work of maintenance departments.

\section{Conclusions}

This paper highlights intelligence as fundamental to the transformation of an eMaintenance system into an iMaintenance system. eMaintenance is accepted worldwide as an ICT supported information system. There are many well-known eMaintenance platforms and frameworks; however, the importance of the intelligence of eMaintenance systems is rarely discussed. 
Since the introduction of eMaintenance, most research has insisted on the relevance of the underlying Information and Communication Technologies infrastructure. By reviewing current eMaintenance conceptual approaches and realizations, this paper argues for the importance of ICT within eMaintenance projects and literature. Intelligence is a fundamental dimension of eMaintenance's growth into an i(ntelligent)Maintenance system.

As a contribution towards the creation of an intelligence-based eMaintenance conceptual framework, the paper proposes modelling the eMaintenance system as an intelligent system. The proposed architecture generates new knowledge on two hierarchical levels by merging CMMS with the CM system. The architecture decomposes plant decisions into two layers (shop floor level and managerial). While connectivity is an essential part of the eMaintenance platform, such decisions are the desired outcome of the iMaintenance system.

Using iMaintenance results in a shift in reliability; decisions are now based on the mining of many diverse data, and are not evident to humans. Instead of reactive maintenance after a breakdown, the present technology takes a pro-active reliability approach, with preventive schedules and a high degree of planning and scheduling. Predictive maintenance technology and analysis is optimized due to the data integration instead of the high degree of unscheduled maintenance and the decisions consider all surrounding data, not just the physical parameters.

All hierarchies in an organization benefit from an intelligent system. Real bottom line results can be delivered, including increased capacity from existing fixed assets, reduced costs both for production and maintenance, improved on-time delivery of products and reduced inventory. With iMaintenance, the business plan, the maintenance policy and improvement strategy inputs are linked to the actual plant components to generate optimum maintenance.

\section{References}

1. A Tsang, 'Strategic dimensions of maintenance management', Journal of Quality in Maintenance Engineering, Vol 8, No 1, pp 7-39, 2002.

2. M Wiseman, 'E-business and physical asset management', Plant Engineering \& Maintenance, Vol 24, No 6, pp 31-43, 2000.

3. D Galar, P Lamban and L Berges, 'Application of dynamic benchmarking of rotating machinery for eMaintenance', International Journal of Systems Assurance Engineering and Management, Springer, Vol 1, No 3, pp 246-262, 2010.

4. I Hausladen and $\mathrm{C}$ Bechheim, 'EMaintenance platform as a basis for business process integration', Industrial Informatics, INDIN '04. 2004 2nd IEEE International Conference on Industrial Informatics, pp 46-51, 26 June 2004.

5. U Kumar, D Galar, A Parida, C. Stenström and L. Berges. 'Maintenance Performance Metrics: A State of the Art Review', International Conference on Maintenance Performance measurement and Management, MPMM, Luleå, 2011.

6. A Muller, A Crespo and B Iung, 'On the concept of eMaintenance : Review and current research’, Reliability Engineering \& System Safety, Vol 93, No 8, pp 11651187, August 2008. 
7. R Karim, 'A Service-Oriented Approach to eMaintenance of Complex Technical Systems', Doctoral Thesis, Luleå University of Technology, Sweden, 2008.

8. EB, 'Encyclopedia Britannica (EB)', http://www.britannica.com/, Accessed on 20 April 2012.

9. O Candell, R Karim, P Söderholm and U Kumar, 'Service-oriented information logistics as support to intelligent transport services', 1 6th World Congress and Exhibition on Intelligent Transport Systems 2009, pp 21-25, Stockholm, Sweden, September 2009.

10. EN 13306:2001, (2001) Maintenance Terminology. European Standard. CEN (European Committee for Standardization), Brussels, 2006.

11. A Crespo, 'On the Definition of Maintenance Management', In The Maintenance Management Framework, Springer Series in Reliability Engineering, Springer London, pp 3-10, 2007.

12. M Barratt, 'Using information technology to improve asset management', http://www.skf.com/portal/skf/home/aptitudexchange?contentId=0.237932.237933. 237936.237942.238543, 2004. Accessed on 11 March 2012.

13. A Labib, 'A decision analysis model for maintenance policy selection using a CMMS', Journal of Quality in Maintenance Engineering, Vol 10, No 3, pp 191202, 2004.

14. A Arnaiz, B Iung, A Adgar, T Naks, A Tohver, $\mathrm{T}$ Tommingas and $\mathrm{E}$ Levrat, 'Information and Communication Technologies Within EMaintenance ', EMaintenance , edited by K Holmberg, E Jantunen, A Adgar, J Mascolo, A Arnaiz, S Mekid, Springer London Ltd, UK, pp 39-60, 2010.

15. MIMOSA, An Operations and Maintenance Information Open System Alliance, http://www.mimosa.org, Accessed on 11 March 2012

16. L Déchamp, A Dutech, $\mathrm{T}$ Montroig, $\mathrm{X}$ Qian, D Racoceanu, I Rasovska, B Brézillon, F Charpillet, J-Y Jaffray, N Moine, B Morello, S Müller, G Nguengang, N Palluat and L Pelissier, 'On the Use of Artificial Intelligence for Prognosis and Diagnosis in the PROTEUS EMaintenance platform', In Proc. of Int. Conf. on Mechatronics and Robotics, (MECHROB'04), Aachen, Deutchland, 2004.

17. B Iung, E Levrat, A Crespo and H Erbe, 'Conceptual framework for eMaintenance : Illustration by eMaintenance technologies and platforms', Annual Reviews in Control, Vol 33, No 2, pp 220-229, December 2009.

18. J Lee, J Ni, D Djurdjanovic, H Qiu and H Liao, 'Intelligent prognostics tools and eMaintenance ', Computers in Industry, Special issue on eMaintenance , Vol 57, No 6, pp 476-489, August 2006.

19. T Han and B Yang: 'Development of an eMaintenance system integrating advanced techniques’, Computers in Industry, Vol 57, No 6, pp 569-580, 2006.

20. C S Byington, M J Roemer and T R Galie, 'Prognostic enhancements to diagnostic systems for improved condition-based maintenance', Proceedings of the IEEE Aerospace Conference, Big Sky, Montana, USA, 2002.

21. C Cempel, H G Natke and J T P Yao, 'Symptom reliability and hazard for systems condition monitoring', Mechanical Systems and Signal Processing, Vol 14, No 3, pp 495-505, 2000.

22. D Galar and A Morant, 'Integration of Production Data in CM for Non-stationary Machinery: A Data Fusion Approach', Condition Monitoring of Machinery in Non- 
Stationary Operations, Proceedings of the second international conference CMMNO'2012, Springer, 2012.

23. D Galar, A Gustafson, B Tormos and L Berges, 'Maintenance decision making based on different types of data fusion', Eksploatacja i niezawodnosc Maintenance and Reliability, Vol 14, No 2, pp 135-144, 2012.

24. A Muller, M-C Suhner and B Iung, 'Maintenance alternative integration to prognosis process engineering', Journal of Quality in Maintenance Engineering, Vol 13, No 2, pp 198-211, 2007.

25. A Muller, M-C Suhner and B Iung, 'Proactive maintenance for industrial system operation based on a formalised prognosis process', Reliability Engineering System Safety, Vol 93, No 2, pp 234-253, 2008. 